\title{
Estudio Experimental de la Tensión Interfacial Transitoria para Sistemas Alcanos-Agua-Triton X-100 empleando la Técnica de la Gota Emergente
}

\author{
Susana Zeppieri, Yurimia Ramírez, Jessica Molina y Aura L. López de Ramos \\ Universidad Simón Bolívar, Departamento de Termodinámica y Fenómenos de Transferencia, \\ Grupo de Fenómenos de Transporte, Apartado Postal 89.000, Caracas 1080-A, Venezuela \\ (e-mail: alopez@usb.ve, zeppieri@usb.ve)
}

\begin{abstract}
Resumen
El objetivo de este trabajo fue estudiar el efecto de la concentración de un surfactante no iónico en agua sobre la tensión interfacial de una serie de alcanos. Para este propósito se seleccionaron el Triton X-100 y los alcanos: hexano, heptano, octano, decano, undecano y dodecano. Las pruebas se realizaron a una concentración $10^{-3}, 10^{-4}$ y $10^{-5} \mathrm{~mol} / \mathrm{L}$ y se empleó el método de la gota emergente para el cálculo de la tensión interfacial. Se observó que la tensión interfacial de las soluciones de Triton X-100 en agua decrece a medida que la concentración de surfactante en solución aumenta. Se concluyó que el valor de la tensión interfacial es función de la concentración, del tiempo de exposición de la interfase con la disolución y del volumen inicial de la gota al momento de iniciar la medida.
\end{abstract}

Palabras clave: Triton X-100, alcanos, método de la gota emergente, tensión interfacial transitoria

\section{Experimental Study of Transient Interfacial Tension for Alkane-Water-Triton X-100 System using the Emergent Drop Technique}

\begin{abstract}
The objective of this work was to study the effect of the concentration of a non-ionic surfactant in water on the interfacial tension of an alkane series. For this purpose Triton X-100 and the alkanes: hexane, heptane, octane, decane, undecane and dodecane were selected for analysis. The tests were done at concentrations of $10^{-3}, 10^{-4}$ and $10^{-5} \mathrm{~mol} / \mathrm{L}$ and the emergent drop model was employed for the calculation of the interfacial tension. In this work it was observed that the interfacial tension of the Triton X-100 solutions in water decreased as the concentration of the surfactant increase. It was concluded that the interfacial tension value is a function of the concentration, the exposure time of the interface with the dissolution and the initial volume of the drop at the moment when the measurement starts.
\end{abstract}

Keywords: Triton X-100, alkanes, emergent drop method, transient interfacial tension 


\section{INTRODUCCION}

El uso de surfactantes juega un rol fundamental en muchos procesos industriales tales como la recuperación del petróleo, como estabilizantes en la Orimulsión ${ }^{\circledR}$ para evitar la coalescencia de las gotas, en la formación y flotación de espumas (Chanamai et al., 2002), en el estudio de la estabilidad de emulsiones (Binks et al., 2000) y microemulsiones (Sagisaka et al., 2007), entre otros. En la actualidad los surfactantes son empleados como agentes inhibidores en la formación de hidratos durante el transporte del gas natural (Chang et al., 2004). El estudio del comportamiento de los surfactantes ha cobrado vital importancia, razón por la cual se han desarrollado técnicas novedosas que son aplicadas para la medición de propiedades físicas de sistemas que involucran a los mismos (Gaudio et al., 2008; Karasawa et al., 2007), tales como la tensión interfacial, a través de la cual se pueden estudiar los procesos de adsorción (enriquecimiento de un componente en la interfase en comparación con el interior de la fase) que ocurren para este tipo de sistemas a nivel de la interfase generada. En estudios anteriores se han determinado la tensión interfacial de sistemas alcano-agua y obtenido correlaciones para el cálculo de la tensión en función de la temperatura y el número de átomo de carbono (Zeppieri et al., 2001). También se han realizado estudios empleando crudo y disolución de surfactante en soluciones salinas con el fin de analizar la influencia de las sales sobre el efecto tensoactivo del surfactante (Ghannam, 2008).

Se han desarrollado múltiples investigaciones en dicha área que han consistido en la mejora de las técnicas de medición, estudio de adsorción-desorción del surfactante en la interfase y de la tensión interfacial transitoria (Fainerman et al., 2009; Fu et al., 2008; Gorevski et al., 2008; Li et al., 2007; Miller et al., 1994; Campanelli y Wang, 1999; Fuentes y López de Ramos, 2001; Horozov y Arnaudov, 1999; Liggieri et al., 1995; MacLeod y Radque, 1993).

Liggieri et al. (1995), realizaron el estudio de la cinética de adsorción del Triton X-100 en una interfase de n-hexano/agua para concentraciones menores a la concentración micelar crítica (concentración a partir de la cual el surfactante forma micelas), aplicando el método de presión capilar para la medición de la tensión interfacial. Concluyeron que la resistencia debida a la cinética de la adsorción era despreciable y que el proceso estaba controlado por la difusión. Además resaltaron que la tensión interfacial era función directa del envejecimiento del medio.

También se han realizado pruebas que han consistido en el estudio de la variación de la tensión interfacial con el tiempo (tensión interfacial transitoria) en mezclas de dos surfactantes (Triton X-100, ácido palmítico y Span 80) en interfases de agua/hexadecano y agua/aceite mineral por debajo de la CMC (Campanelli y Wang, 1999), utilizando el método del tensiómetro de volumen de la gota. En este estudio se encontró que la adsorción cinética dominó en la mezcla ácido palmítico con Triton X100 y que el coeficiente de difusión aparente era independiente de la presencia del acido palmítico. Mientras que para la mezcla de Span 80/Triton X-100 la adsorción cinética estuvo influenciada por los dos surfactantes y en este caso los cambios relativos de concentración de surfactante afectaron la tensión interfacial transitoria del sistema.

El trabajo que a continuación se presenta tiene por objeto el estudio del efecto de la concentración de una disolución de surfactante no iónico (Triton X-100) en agua sobre la tensión interfacial transitoria en diversos alcanos, tales como hexano, heptano, octano, decano, undecano y dodecano, mediante la Técnica de la Gota Colgante (Fuentes y López de Ramos, 2001). Se seleccionaron estos alcanos porque son hidrocarburos intermedios que podrían tener comportamiento similar al del petróleo $y$, de esta manera, se podrían utilizar estos resultados en el desarrollo de nuevas tecnologías en recuperación mejorada de crudos por medio de la inyección de surfactante.

\section{MODELO DE LA GOTA EMERGENTE}

Para la determinación de la tensión interfacial de los sistemas de hidrocarburos Triton X-100/agua se empleó el Método de la Gota Emergente que es una variante del Modelo de la Gota Colgante.

El método de la gota colgante permite la determinación de la tensión superficial a partir del cálculo de diversos factores de forma que caracterizan el perfil de una gota. Para el cálculo de la tensión 
superficial se requiere de la resolución de la ecuación de Laplace-Young, que tiene una forma particular para el caso de una gota que cuelga verticalmente (Fig. 1), y que se muestra a continuación:

$$
2 H=-\frac{\Delta \rho g}{\sigma} y+\frac{2}{R_{o}}
$$

donde $\Delta \rho$ es la diferencia de densidades entre la fase líquida y la gaseosa y $R_{0}$ es el radio de curvatura en $\mathrm{y}=0$.

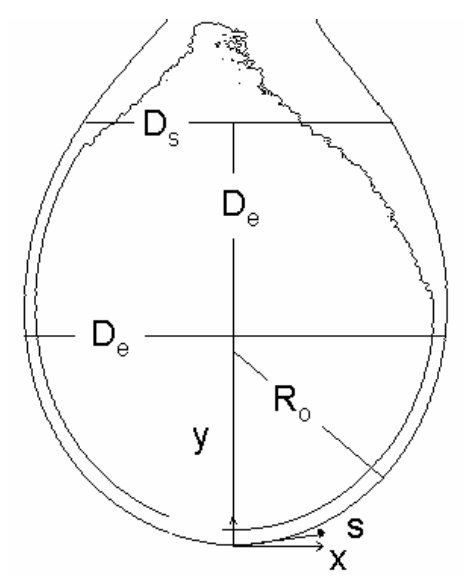

Fig. 1: Gota y parámetros geométricos.

$\mathrm{H}$ se refiere a la curvatura promedio de la gota que es función de y expresándose de la siguiente manera:

$2 H=\frac{y_{x}}{x\left(1+y_{x}^{2}\right)^{1 / 2}}+\frac{y_{x x}}{\left(1+y_{x}^{2}\right)^{3 / 2}}$

donde $y_{x} y y_{x x}$ se refieren a la primera y segunda derivadas de y con respecto a $x$. Si se trabaja la ecuación de Laplace-Young tal y como se presenta en la Ec. (2), se encuentra que pueden ocurrir indeterminaciones matemáticas a la hora de la resolución de la ecuación en el punto ecuatorial de la gota. Para eliminar dichas indeterminaciones se acostumbra a trabajar la Ec. (1) según el siguiente cambio de variable, referido al arco de longitud (s):

$\operatorname{sen} \theta=\frac{d y}{d s}$ y que, $\cos \theta=\frac{d x}{d s}$

sustituyendo la primera y segunda derivadas en las Ec. (2) y (3), se obtiene:

$$
\begin{aligned}
& \frac{d \theta}{d S}=2-\beta Y-\frac{\operatorname{sen} \theta}{X} \\
& \frac{d Y}{d S}=\operatorname{sen} \theta \quad \frac{d X}{d S}=\cos \theta \quad \beta=\frac{\Delta \rho g R_{o}{ }^{2}}{\sigma}
\end{aligned}
$$

donde,

$Y=\frac{y}{R_{o}} \quad X=\frac{x}{R_{o}} \quad S=\frac{S}{R_{o}}$ 
El sistema de ecuaciones (4) y (5) se resuelve con un método numérico. Una vez generados los perfiles de la gota colgantes, se procede a calcular $D_{e}, D_{s}$ y $R_{o}$ (Fig. 1). Para determinar los valores de estas variables geométricas, diferentes secciones de la gota se ajustan mediante polinomios de segundo y tercer orden: la parte baja de la gota para $R_{0}$, la zona ecuatorial para $D_{e}$ y la zona cercana al cuello para $D_{s}$ (Fariñas et al., 1998).

\section{PROCEDIMIENTO EXPERIMENTAL}

El método experimental consistió de:

Productos químicos utilizados: Las disoluciones de surfactante en agua utilizadas fueron preparadas con Triton X-100 extra puro de laboratorios Aldrich y con agua destilada obtenida de un destilador de laboratorio marca Corgning (modelo ag-11). El hexano de laboratorios MERCK, el heptano y undecano de 99+\% HPLC grado de pureza de SIGMA-ALDRICH, el octano 99+\% anhidro de laboratorios ALDRICH®, el decano y dodecano de Fisher Scientific con 99+\%.

Preparación de las disoluciones: Se prepararon tres concentraciones $\left(10^{-3}, 10^{-4}\right.$ y $\left.10^{-5} \mathrm{~mol} / \mathrm{l}\right)$ de mezcla Triton X-100 en agua. Inicialmente se tomó una muestra de Triton X-100 con una pipeta de $20 \mathrm{ml}$ y se pesó una gota de Triton X-100. Para dicho valor en peso se calculó analíticamente el volumen de agua requerido para alcanzar la concentración de mezcla a preparar. Seguidamente la disolución fue sometida a agitación en un agitador de vibración Chemepec, por un intervalo de tiempo que varió de 40 min a 5 horas según fuera la concentración de Triton X-100 a preparar, con el fin de lograr una muestra uniforme, es decir cuya tensión interfacial fuera la misma en todo el volumen de la muestra. Luego la muestra se dejó reposar unas horas para esperar que la espuma generada por la agitación desapareciera.

Densidad de las sustancias utilizadas: Para las disoluciones de Triton X-100 en agua se calculó la densidad según la proporción molar de cada uno de los componentes. Mientras que para los alcanos utilizados se calculó la densidad según la siguiente ecuación (Perry, 1984):

$$
\begin{aligned}
& \rho=\frac{P_{c}}{\left.T_{c} R Z_{c}{ }^{\left[1+\left(1-T_{r}\right)^{2 / /}\right]}\right]}\left[1+\frac{9 Z_{c} N\left(P-P_{\text {sat }}\right)}{P_{c}}\right]^{1 / 9} \\
& N=\left[1-0,89 \omega^{1 / 2}\right]\left[\exp \left(6,9547-76,2853 T_{r}+191,3060 T_{r}^{2}-203,5472 T_{r}^{3}+82,7631 T_{r}^{4}\right)\right]
\end{aligned}
$$

donde $P_{c}, T_{c}$ y $Z_{c}$ son las constantes críticas propias de cada sustancia, $\omega$ es el factor acéntrico y $T_{r}$ es la temperatura reducida a la cual se realizó el experimento, $\mathrm{P}_{\text {sat }}$ es la presión de saturación.

Modelo de la Gota Emergente: El equipo que se utilizó para crear el sistema de la gota de alcano sumergida en la disolución de surfactante en agua consistió de una celda de visualización de acero inoxidable (Fig. 2) diseñada para trabajar a condiciones de $340 \mathrm{~atm}$ y $100{ }^{\circ} \mathrm{C}$, dicha celda está constituida por dos paredes removibles con visores que sirven para captar la imagen de la gota y que además ajustan dos cristales de zafiro de 25,05 mm de diámetro que están protegidos cada uno por un par de empacaduras de goma, que garantizan la retención de los líquidos de trabajo. La gota emergente de alcano se introdujo en la celda por medio de una jeringa de vidrio marca Becton, Dickinson de $3 \mathrm{ml}$, a la cual se le adaptó una aguja truncada de $0,45 \mathrm{~mm}$ de diámetro interno y se fijó a la parte inferior de la celda con un conector de cobre NPT 1/8". La disolución se introdujo lateralmente a través de un sello de goma, utilizando una inyectadora desechable de $5 \mathrm{ml}$. Una vez realizada cada una de las experiencias experimentales se lavó cuidadosamente todo el equipo utilizado a fin de evitar contaminación para experiencias posteriores. Todo el sistema de medición se encuentra sobre una mesa óptica con patas hidráulicas para eliminar vibraciones mecánicas que puedan desprender la gota del capilar. 


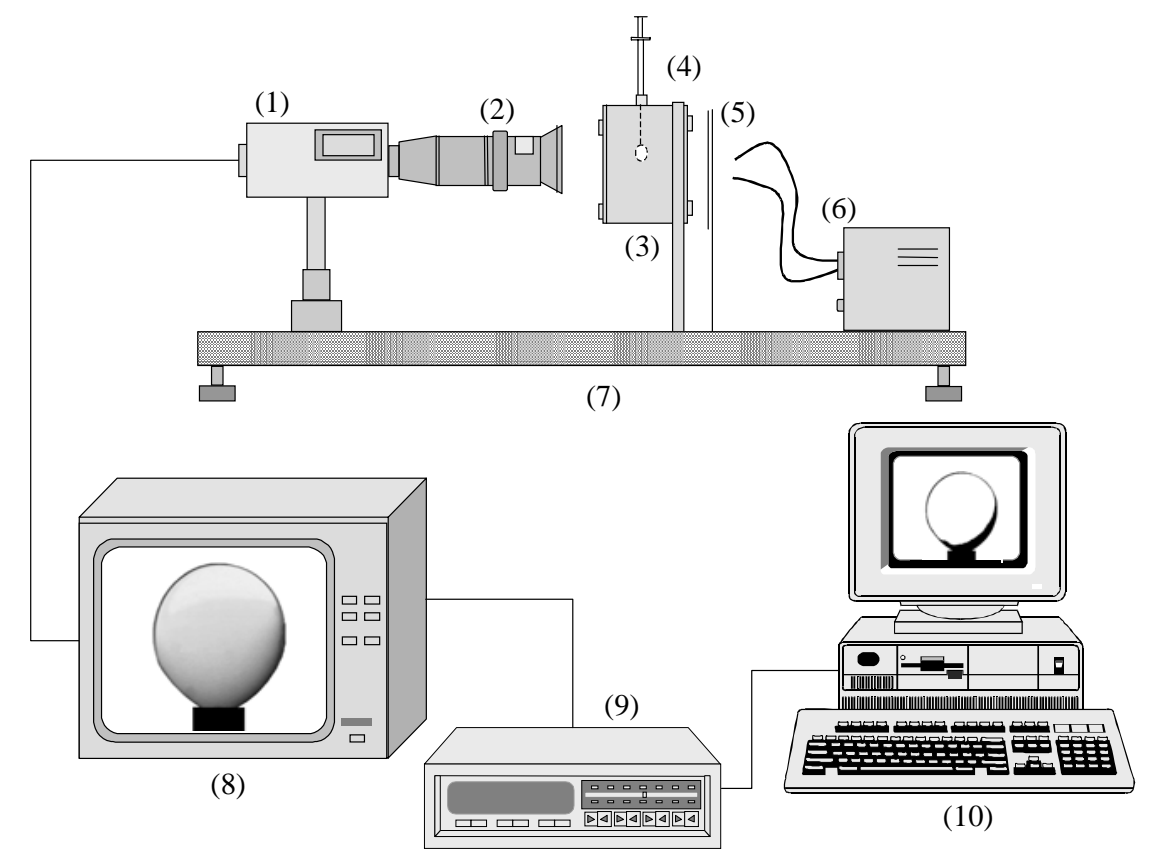

Fig. 2: Equipo experimental (1: video camera, 2: lente, 3: celda de visualización, 4: aguja, 5: difusor, 6: lámpara de fibra óptica, 8: monitor de alta resolución, 9: video grabadora y 10: computador con tarjeta Targa).

Con la finalidad de que los resultados se vieran afectados por el medio lo menos posible, se conectó la celda a un baño termostático modelo HAAKE A81, que garantizó que la temperatura se mantuviera constante y que pudo ser leída en un termómetro digital Fluke $52 \mathrm{~K} / \mathrm{J}$.

Para la captura de la imagen se colocó en la parte posterior de la celda una pantalla difusora de papel vegetal y luego se iluminó con una lámpara. Frente a la parte delantera de la celda se encuentra ubicado un sistema de rieles que permite el ajuste en tres direcciones de la cámara de video (CCD-72) a la cual se le adapta un lente Nikkon 55mm con un anillo de extensión PK-13. Dicha cámara estaba conectada a un monitor Sony (PVM-2030) que permitió la visualización de la gota formada y que fue grabada por una video grabadora VHS Philips VR79950.

Procesamiento de imágenes: Una vez grabadas las gotas, se procede a la digitalización de las imágenes para obtener el perfil x'y de la gota y luego introducir estos datos en el programa DROP 98, que una vez introducidos los datos de escalamiento y seleccionado como modo de ajuste el de factor de forma el programa dará como resultados para cada gota los valores de diámetro ecuatorial $\left(D_{e}\right)$ en $\mathrm{mm}$, diámetro de cuello $\left(D_{\mathrm{S}}\right)$ en $\mathrm{mm}$, radio de curvatura $\left(R_{0}\right)$ en $\mathrm{mm}$ y la tensión interfacial $(\sigma)$ en $\mathrm{mN} / \mathrm{m}$.

\section{RESULTADOS Y DISCUSIÓN}

Tensión superficial de mezclas de Triton X-100 en agua a diferentes concentraciones

En la Fig. 3 se puede observar que el comportamiento de la tensión superficial fue decayendo en la medida que aumentó la concentración de Triton X-100 en agua.

A bajas concentraciones el valor de tensión superficial es bastante parecido al del agua mientras que a altas concentraciones se observa el efecto reductor en el valor de tensión superficial que ejerce el surfactante. Para calcular el valor de la concentración micelar crítica (CMC) se determinó la tensión superficial variando la concentración de Triton X-100 en agua. El valor de CMC obtenido fue de $1,8 \times 10^{-4} \mathrm{~mol} / \mathrm{l}$, el cual se encuentra entre los valores reportados en la literatura. Liggieri et al. (1995), reportan $1,17 \times 10^{-4} \mathrm{~mol} / \mathrm{l}$ y Wu et al. (1999) obtienen el valor de $3 \times 10^{-4} \mathrm{~mol} / \mathrm{l}$. 


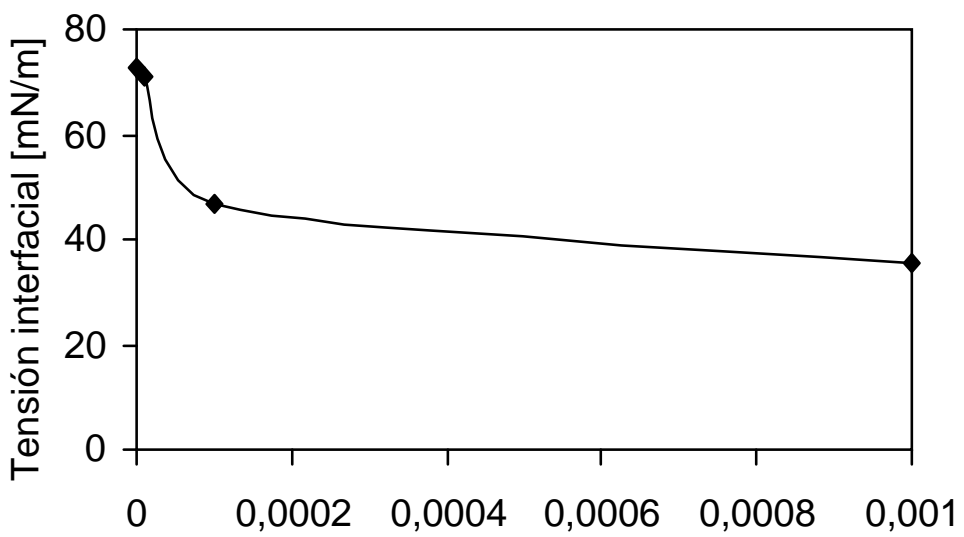

Concentración de Tritón X-100 [mol/l]

Fig. 3: Tensión interfacial de mezclas de distintas concentraciones de Triton X-100 en agua.

\section{Alcanos en diferentes concentraciones de Triton X-100 en agua.}

En general se observó que la tendencia de la tensión interfacial para todas las concentraciones fue la de disminución en el tiempo, poniéndose en evidencia con el cambio de la forma de la gota (Fig. 4), es decir, con la variación de la curvatura de la interfase líquido-líquido.

De la secuencia de imágenes mostrada en la Fig. 4 puede observarse que además de la disminución de la curvatura de la gota, está cambiando también su volumen. Este cambio se debe al proceso de transferencia de masa que ocurre a través de la interfase de la gota. Esta transferencia de masa de la gota hacia el medio puede deberse a que el Triton X-100 adsorbido en la interfase, que es parcialmente soluble en el alcano, oriente su parte hidrofóbica, de forma tal de que encierre la molécula de alcano y la arrastre hacia el seno del fluido (medio continuo).

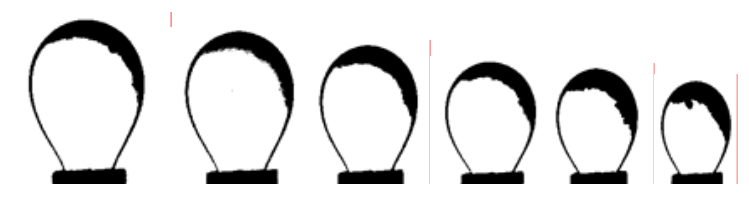

Fig. 4: Secuencia de reducción del tamaño de una gota de hexano a través del tiempo, por el efecto del surfactante.

Al inicio, en todos los casos estudiados, se observó una caída pronunciada en el valor de la tensión interfacial (Fig. 5a, 5b, 5c, 5d, 5e, 5f). Esto puede deberse a la acción del surfactante en la interfase durante los primeros segundos de exposición, en donde el gradiente de concentración entre el bulto y la interfase es grande, debido a la poca saturación inicial del surfactante en la misma. A medida que el tiempo de exposición de la interfase aumenta el sistema tiende al equilibrio.

Para cada sistema estudiado se formaron seis gotas diferentes con el fin de verificar la repetibilidad de los experimentos. La desviación obtenida para la concentración de $10^{-5}$ y $10^{-4}$ fue de $3 \%$, mientras que para $10^{-3}$ fue de $5 \%$.

El tiempo máximo de experimentación de una gota está limitado por el tamaño de la misma, debido a que el modelo matemático utilizado en este trabajo requiere de la existencia del diámetro de cuello $\left(D_{s}\right)$. En el trabajo realizado por Touhami et al. (1996), relacionado con la tensión transitoria, el tiempo máximo de medición estaba restringido por el desprendimiento de la gota. La gota se desprendía del capilar debido a que las fuerzas de adhesión entre el fluido y el sólido se reducía por efecto del tensoactivo y esto originaba el desprendimiento de la gota del capilar. Este efecto no ocurre en este trabajo ya que el radio del capilar seleccionó con el fin de obtener una gota estable. 


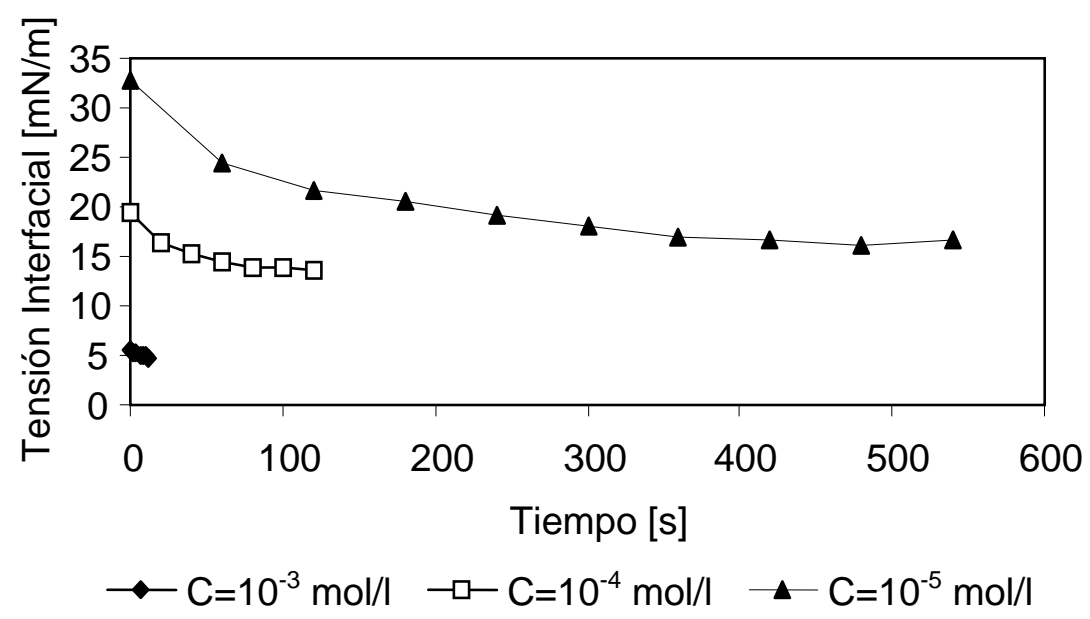

Fig. 5a: Tensión interfacial transitoria del hexano en diferentes concentraciones de Triton X-100 en agua.

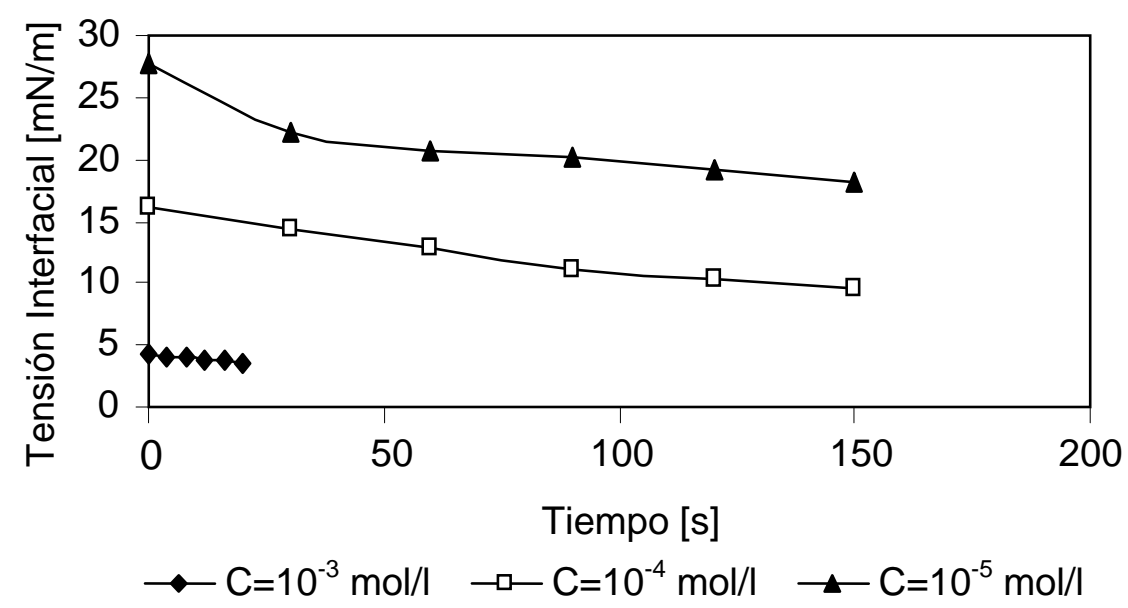

Fig. 5b: Tensión interfacial transitoria del heptano en diferentes concentraciones de Triton X-100 en agua.

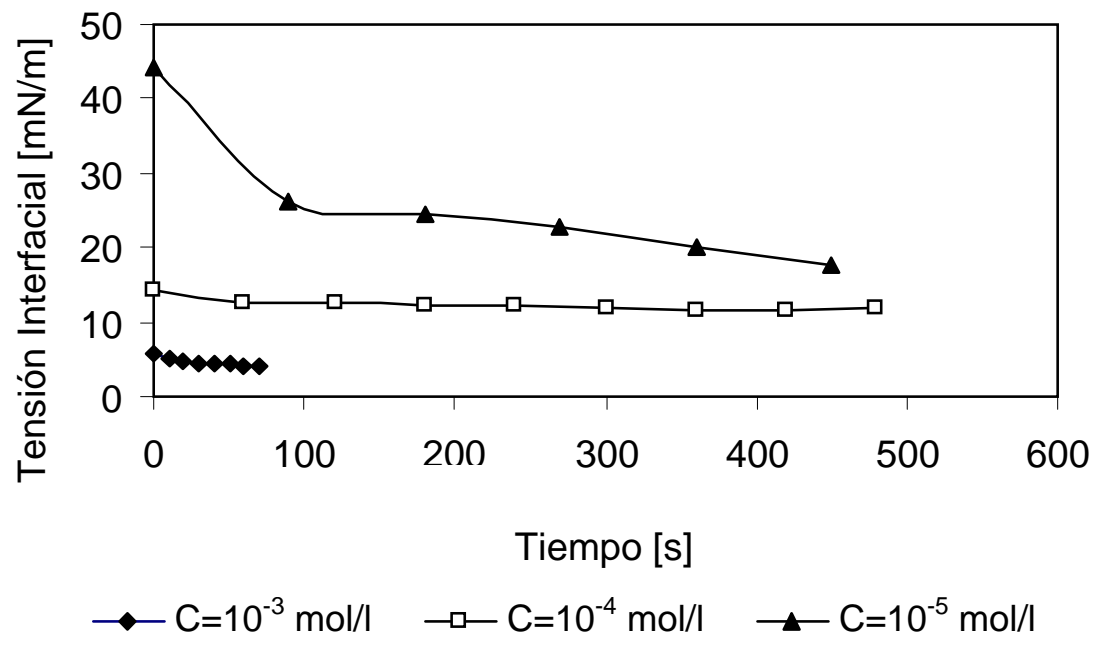

Fig. 5c: Tensión interfacial transitoria del octano en diferentes concentraciones de Triton X-100 en agua. 


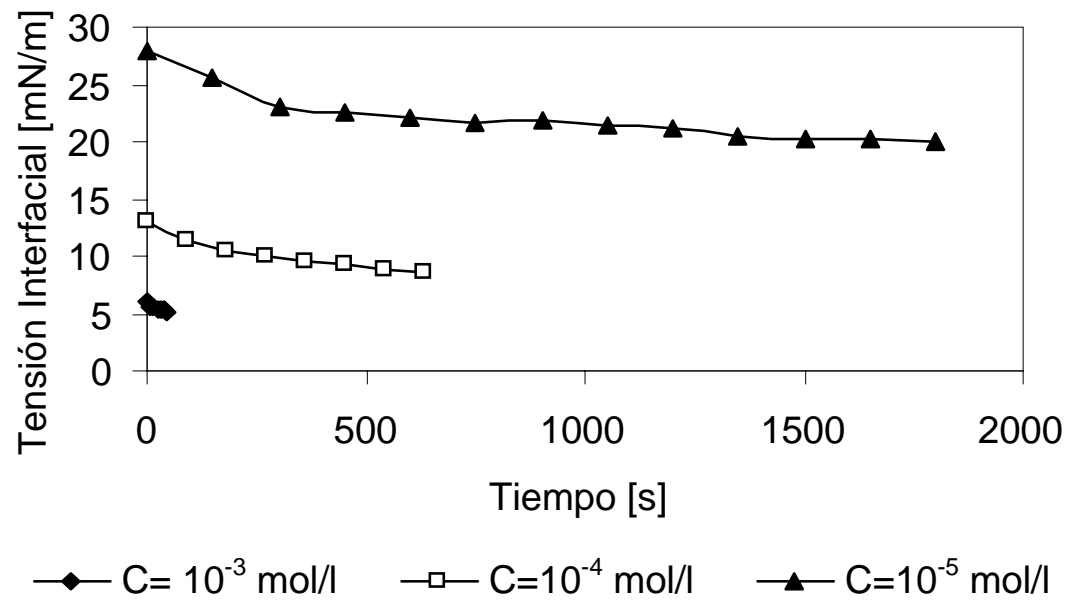

Fig. 5d: Tensión interfacial transitoria del decano en diferentes concentraciones de Triton X-100 en agua.

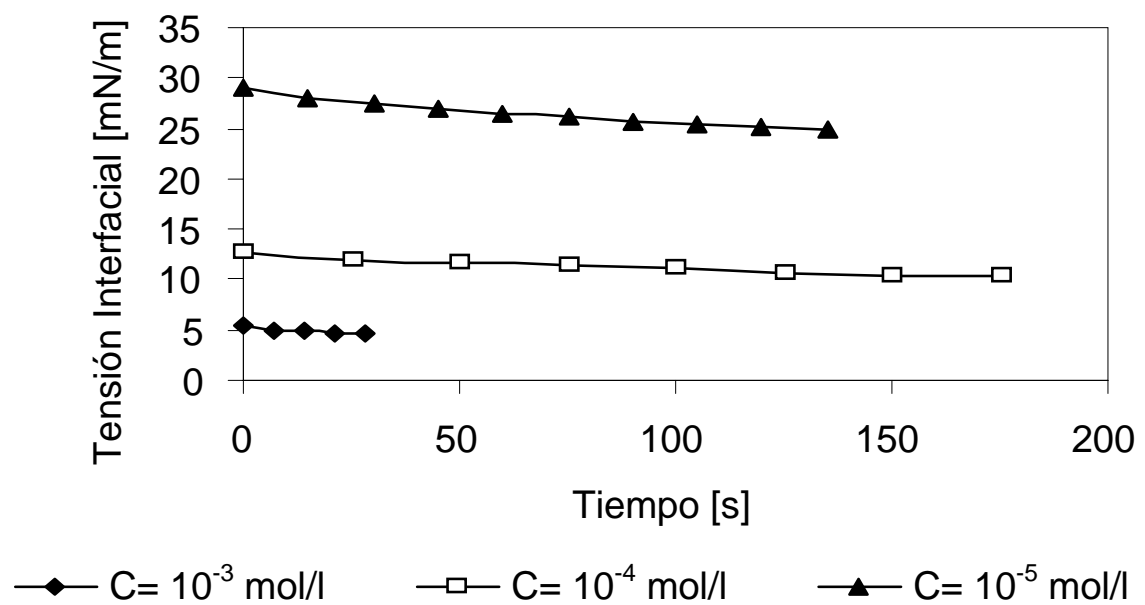

Fig. 5e: Tensión interfacial transitoria del undecano en diferentes concentraciones de Triton X-100 en agua.

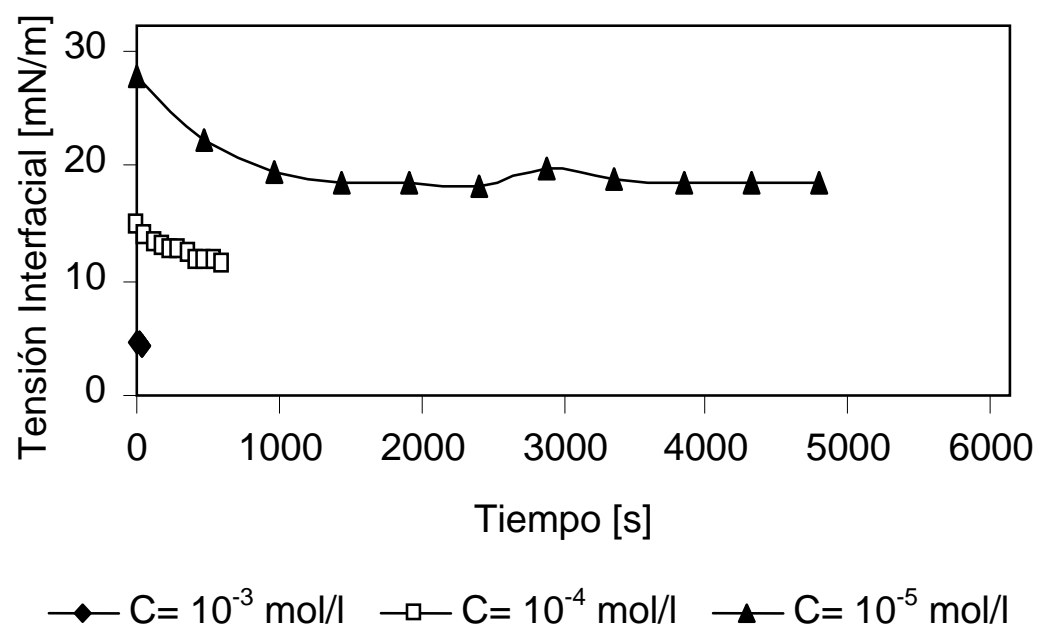

Fig. 5f: Tensión interfacial transitoria del dodecano en diferentes concentraciones de Triton X-100 en agua. 
En la medida que la concentración de la disolución de surfactante en agua fue aumentando, el tiempo que duró el efecto del surfactante sobre la tensión interfacial del alcano fue disminuyendo, tal como se aprecia en las Figs. 5. También se observa que si el medio tiene menor concentración de Triton X-100, la tensión interfacial se mantiene en un rango de valores mayores. El cambio del valor de la tensión interfacial y del tiempo de estabilización del sistema está relacionado con el proceso de adsorción - desorción del surfactante en la interfase. A medida que el surfactante se adsorbe en la interfase la película se debilita cambiando las fuerzas que actúan sobre la interfase.

\section{Comportamiento de los alcanos a la misma concentración.}

En la Fig. 6 se observa que para el caso de concentración de $10^{-3} \mathrm{~mol} / \mathrm{l}$, el hexano y el octano se manejan en un rango de tensiones interfaciales similares a pesar de que existe una marcada diferencia entre el tiempo de acción del surfactante y los valores finales de tensión interfacial; mientras que en el heptano se obtienen los menores valores de la tensión interfacial transitoria, lo cual probablemente esté asociado con interacciones o rearreglos que se estén dando a nivel molecular para el caso particular del heptano. Este mismo comportamiento se obtiene para las concentraciones de Triton X-100 en agua de $10^{-4} \mathrm{~mol} / \mathrm{l}$ y $10^{-5} \mathrm{~mol} / \mathrm{l}$.

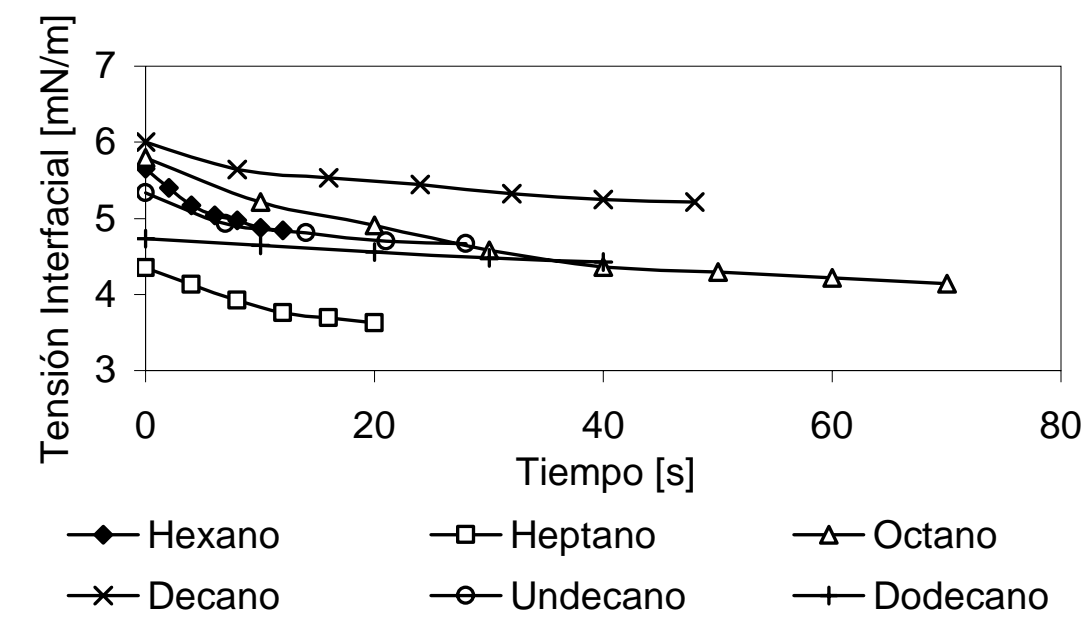

Fig. 6: Comparación del efecto en la tensión interfacial entre los diferentes alcanos con Triton X 100/agua a $10^{-3} \mathrm{~mol} / \mathrm{l}$.

\section{CONCLUSIONES}

El valor de tensión superficial de las disoluciones a diferentes concentraciones de Triton X-100 en agua va disminuyendo en la medida que la concentración de surfactante va aumentando. La concentración micelar crítica (CMC) para la disolución de Triton X-100 en agua es de 1,8×10-4 mol/l.

Para los sistemas de alcanos estudiados hubo una reducción significativa del valor de tensión interfacial en el tiempo, a medida que la concentración de surfactante aumentó, disminuyó el tiempo en el cual se alcanzó la tensión interfacial del equilibrio ya que el proceso de adsorción en la interfase depende de la concentración de surfactante en el medio. Pero en la medida que la concentración del medio fue menor, el porcentaje de reducción con respecto a la tensión interfacial inicial del alcano tendió a ser mayor.

En el caso del heptano se alcanzan los valores más bajos de tensión interfacial para las concentraciones de $10^{-3} \mathrm{y}$ de $10^{-5} \mathrm{~mol} / \mathrm{l}$.

El valor de la tensión interfacial es función de la concentración de surfactante en el medio, el cual depende del tiempo de exposición de la interfase con la disolución y del volumen inicial de la gota al momento de iniciar la medida. 


\section{REFERENCIAS}

Binks, B. P., W. Cho, P.D. Fletcher y D.N. Petsev; Stability of oil-in-water emulsions in a low interfacial tension system, Langmuir: 16, 1025-1034 (2000).

Campanelli, J.R., y X.J. Wang; Dynamic interfacial tension of surfactant mixtures at liquid-liquid interfaces, J. Colloid Interface Sci.: 213, 340-351 (1999).

Chanamai, R., G. Horn y D. McClements; Influence of oil polarity on droplet growth in oil-in-water emulsions stabilized by a weakly adsorbing biopolímero or a nonionic surfactante, J. Colloid and Interface Sci.: 247, 167-176 (2002).

Chang-Yu, S., C. Gunag-Jin y Y. Lan-Ying; Interfacial tension of methane+water with surfactant near the hydrate formation conditions, J. Chem. Eng. Data: 49, 1023-1025 (2004).

Fainerman, V. B., y otros seis autores; Adsortion layer characteristics of Triton surfactants4. Dynamic surface tension and dilational visco-elasticity of micellar solutions, Colloids and Surface A: Physicochem. Eng. Aspect: 334, 22-27 (2009).

Fariñas, J., P. Blanco, J.M. Ledanois y A.L. López de Ramos; Determinación de la tensión superficial mediante digitalización de imágenes de la gota colgante, Información Tecnológica: 9(1), 167-173 (1998).

Fu, S., y otros seis autores; Study on dynamic interfacial tensión of 3-dodecyloxy-2-hydroxypropyl trimethyl ammonium bromide at liquid/liquid interface, Fluid Phase Equilibria: 269, 93-97 (2008).

Fuentes, J. y A. L. López de Ramos; Nuevo método para medir la tensión superficial mediante la técnica de la gota colgante, Información Tecnológica: 12 (2), 181-188 (2001).

Gaudio, L., y otros nueve autores; Dynamic interfacial properties of props. Relevant to W/O-emulsionforming systems: A refined mesurement apparatus, Colloids and Surface A: Physicochem. Eng. Aspect: 323, 3-11 (2008).

Ghannam, M.; Experimental investigation of dynamic interfacial tensiono $f$ crude oil-different aqueous Solutions, J. of Chem. Eng. Of Japan: 41(2), 121-129 (2008).

Gorevski, N., R. Miller y J. K. Ferri; Non-equilibrium Exchange kinetics in sequential non-ionic surfactant adsorption: Theory and experiment, Colloids and Surface A: Physicochem. Eng. Aspect: 323, 12-18 (2008).

Horozov, T. y L. Arnaudov; A novel fast technique for measuring dynamic surface and interfacial tension of surfactant solutions at constant interfacial area, J. Colloid Interface Sci.: 219, 99-109 (1999).

Karasawa, M., T. Hasegawa y T. Narumi; Measurement of dynamic surface tensiono $f$ surfactante solutions, J. of the Society of Rheology: 35(5), 265-271 (2007).

Li, G., S. Prasad y A. Dhinojwala; Dynamic interfacial tension at the oil/surfactant-water interface, Langmuir: 23, 9929-9932 (2007).

Liggieri, L., F. Ravera y A. Passerone; Dynamic interfacial tension measurements by a capillary pressure method, J. Colloid Interface Sci.: 169, 226-237 (1995).

MacLeod, C. A. y C. J. Radke; A growing drop technique for measuring dynamic interfacial tension, J. Colloid Interface Sci.: 160, 435-448 (1993).

Miller, R., P. Joos y V. B. Fainerman, Dynamic studies of soluble adsorption layers, Progr. Colloid Polym. Sci., 97, 188-193, (1994). 
Perry, R. H., D. W. Green y J. O. Maloney; Physical and chemical data, Perry's chemical engineers' handbook, $6^{\text {ta }}$ Edición McGraw-Hill Book Company, 3.273-3.274, New York, EEUU (1984).

Sagisaka, M., y otros nueve autores; Surfactant-mixing effects on the interfacial tension and the microemulsion formation in water/supercritical $\mathrm{CO}_{2}$ systems, Langmuir: 23, 2369-2375 (2007).

Touhami, Y., G.H. Neale, V. Hornof y H. Khalfalah; A modified pendant drop method for transient and dynamic interfacial tension measurement, Colloids and Surface A: Physicochem. Eng. Aspect: 112, 31-41 (1996).

Wu, N., J. Dai y F. J. Micale; Dynamic surface tension measurement with a dynamic Wilhelmy plate technique, J. Colloid Interface Sci.: 215, 258-269 (1999).

Zeppieri, S., J. Rodríguez y A. L. López de Ramos; Interfacial tension of alkane water Systems, J. of Chem. and Eng. Data: 46(5), 1086-1088 (2001). 